\title{
Treatment of Cervical Ligamentum Flavum Calcification with Single-Level Double-Door Laminoplasty
}

\author{
Ya-Jun Liu*, Xiao-Feng LE, Lin HU, Da HE, Sai MA and Wei Tian \\ Department of Spine Surgery, , China
}

*Corresponding author: Ya-jun Liu, Department of Spine Surgery, China

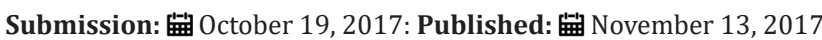

\begin{abstract}
Calcifcation of Ligamentum Flavum (CLF) is an uncommon disorder. We present a 72-year-old patient with cold right extremities and headache. The imaging investigations conclude for the diagnosis of calcification of the ligamentum flavum. Our observation illustrates the difficulties to distinguish the ossification or calcification of the ligament before the operation. Finally, we draw attention to the efficacy of medical treatment in our case and we discuss the different therapies proposed in the literature.
\end{abstract}

Keywords: Ligamentum flavum; Calcification; Laminoplasty

Abbreviations: CLF: Calcifcation of Ligamentum Flavum

\section{Introduction}

CLF is an uncommon disorder. This condition is probably underestimated and most reported cases were Japanese patients. A pub med search found no Chinese case report. The most common presentation of CLF is sub acute myelopathy due to spinal cord compression by the calcifcations in the absence of precipitating factors. CLF in this case occurred in a Chinese patient who presented misleading clinical symptoms.

Case Report

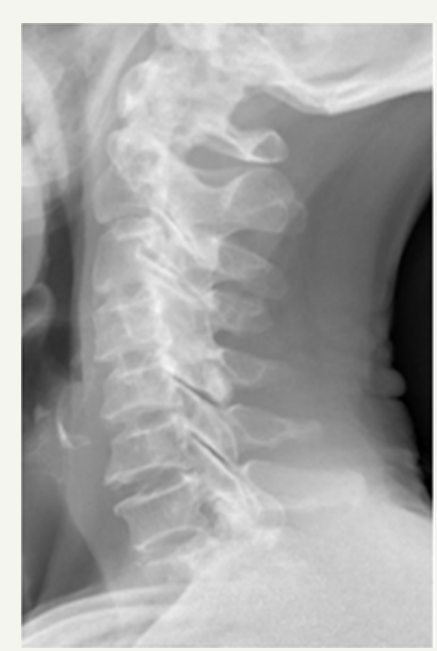

Figure 1: Plain Radiographs of the cervical spine showed no obvious abnormalities.

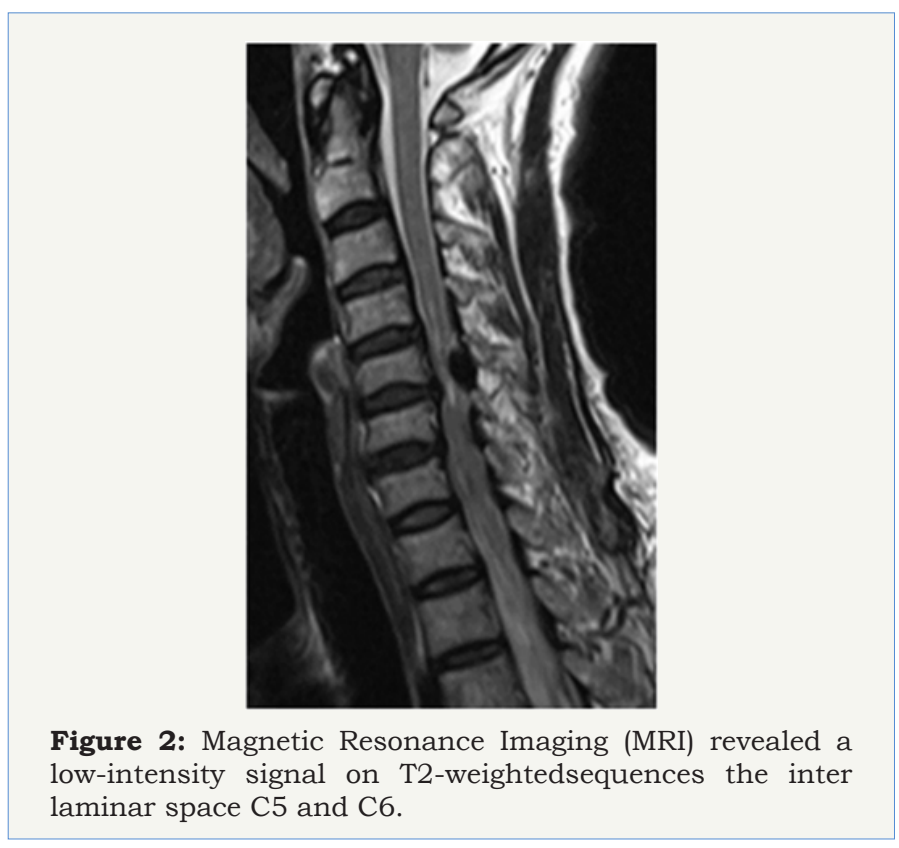

A 72-year-old Chinese woman was admitted with a history of 1year cold right extremities and headache.6 months ago, she complained of walking instability 4 months ago, she suffered from severe pain in the left upper limb, with slight numbness at the fingertips of both hands when coughing. No history of any cardiovascular system diseases was present (Figure 1). At physical 
examination, she showed a stable life signs. The temperature of right extremities was significantly lower than the contralateral side, but the artery pulsatile normal. She had no sensory defcitand no sphincteric involvement. Her limbs muscle strength were grade IV. Knee flexion hyperreflexia, pathological reflex $(+)$, straight foot test $(+)$, cervical JOA score of 8 . Additional blood analysis showed normal (Figure 2). Preoperative diagnosis: cervical spondylotic myelopathy with sympathetic symptoms caused by cervical ligamentum flavum lesions (ossification or calcification).

\section{Surgical management}

Take the posterior incision of C4-6 and expose the lamina. Carefully split the $\mathrm{C} 5$ spinous process with a grinding mill to reveal the thick yellow ligament, carefully remove the lesion. When the dura materis distended, an artificial coral bone is fixed on the spinous process of C5. The lesions were 2 globular gray tissues of peanut size, and microscopic examination showed fibrous cartilage and collagen fibrous tissue with calcification (Figure 3).

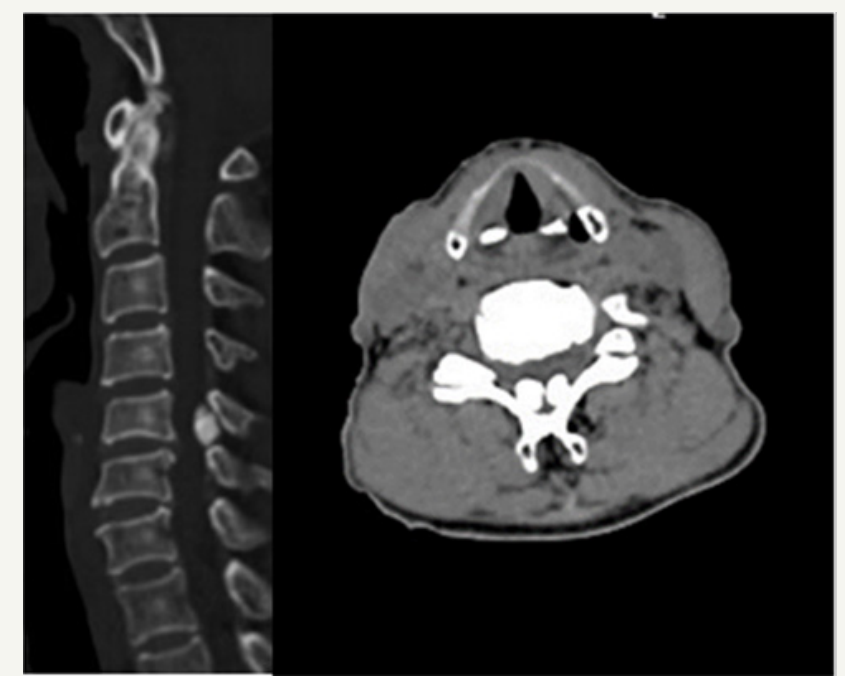

Figure 3: a) Sagittal b) transversal \& Computed Tomography (CT) scan confirmed the presence of bilateral oval ligamentum flavum lesions, localized from C5 to C6.

\section{Results}

1 week after surgery, the patient's right extremities' skin temperature returned to normal. She could walk stably, and her headache and fingertips numbness disappeared. Limb muscle strength was IV +. JOA score was 14 . After 3 months, JOA score was 15. After 6 months, JOA score was 15 points. Up to now 8 months, the patient was asymptomatic.

\section{Discussion}

It is reported in literature that CLF can be treated conservatively with colchicines [1], so it is necessary to distinguish the ossification or calcification of the ligament before the operation. Miyasakaet al. [2] have shown that the ligamentum flavum ossification of the following morphological features: usually involved two or more spinal levels; often associated with ossification of the posterior longitudinal ligament and thickening of the laminae; usually extends along the joint and pedicle laterally $[3,4]$; appeared as a beaklike or mound like bony excrescence arising from the laminae. The calcification: more involved in a single level; lesions mostly spherical nodules; usually both sides appear simultaneously, and lamina adhesion light. Combined with this case, the possibility of CLF was considered preoperatively, and the pathological results confirmed our guess.

Because the patient's pain is unbearable, she strongly required for surgical treatment. There are no reports of single-level doubledoor laminoplasty in the treatment of patients with cervical CLF. Generally, the conclusion that laminoplasty is biologically superior to laminectomy is now recognized by academics, because laminoplasty reduce the possibility of postoperative cervical kyphosis and have lower risk of injury of blood vessels and nerves during the operation [5]. In the past, it was considered that the cervical lesions under 3 segments were suitable for open-door, and over 3 segments were suitable for double-door, but there was controversy. Recently, Hirabayashi et al. [6] have compared the results of cervical open-door and double-door laminoplasty, and summed up the indications. They thought "cervical spondylotic myelopathy combined with bilateral radiculopathy" were more suitable for double-door. The CLF is mostly bilateral compression, so we believe that the CLF is more suitable for double door laminoplasty. We firstly attempted to treat the disease with singlelevel double open-door laminoplasty. The curative effect is good and the patient is satisfied. Maybe this can provide reference for colleagues.

\section{References}

1. Mahmoud I, Tekaya R, Saidane O, Chaabouni L, Zouari R (2012) Original Etiology of Acute and Febrile Neck Pain: Cervical Calcification of Ligamentum Flavum. Orthop Muscul Syst 1(3): 109.

2. Miyasaka K, Kaneda K, Sato S, Iwasaki Y, Abe S, et al. (1983) Myelopathy due to ossification or calcification of the ligamentum flavum: radiologic and histologic evaluations. AJNR Am J Neuroradiol 4(3): 629-632.

3. Yanagi T (1972) Neurologic disorders caused by ossification of the ligamenta flava of the thoracic spine; analysis of $x$-ray picture and diagnostic significance. Rinsho Shinkeigaku 12(11): 562-570.

4. Kodama T, Okubo K, Matsukado Y (1979) Myelopathy due to ossified ligamenta flava in lower thoracic region (author's transl). No Shinkei Geka 7(9): 867-873.

5. Saito Y, Toshihiko T, Kazuo K, Akira H, Shinya K (2001) Comparison of the Results of the Cervical Laminoplasty (Hattori's Method) and the Cervical Laminectomy. Orthopedics \& Traumatology 50(1): 6-8.

6. Hirabayashi S, Yamada H, Motosuneya T, Watanabe Y, Miura M, et al. (2010) Comparison of enlargement of the spinal canal after cervical laminoplasty: open-door type and double-door type. Eur Spine J 19(10): 1690-1694. 